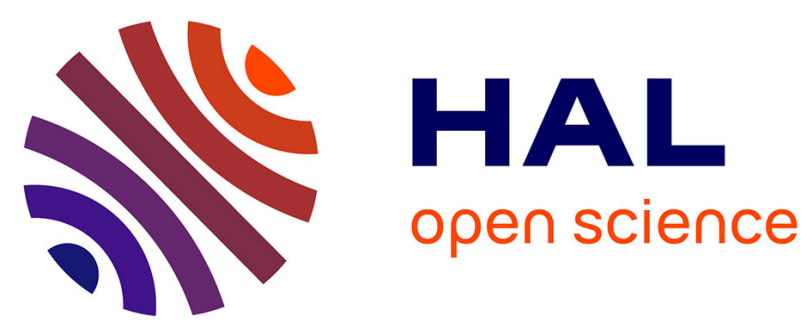

\title{
A Flexible Power Crossbar-based Architecture for Software-Defined Power Domains
}

Francesco Di Gregorio, Gilles Sassatelli, Abdoulaye Gamatié, Arnaud Castelltort

\section{- To cite this version:}

Francesco Di Gregorio, Gilles Sassatelli, Abdoulaye Gamatié, Arnaud Castelltort. A Flexible Power Crossbar-based Architecture for Software-Defined Power Domains. EPE'20 ECCE Europe, Sep 2020, Lyon (virtual), France. hal-02950802

\section{HAL Id: hal-02950802 https://hal.science/hal-02950802}

Submitted on 28 Sep 2020

HAL is a multi-disciplinary open access archive for the deposit and dissemination of scientific research documents, whether they are published or not. The documents may come from teaching and research institutions in France or abroad, or from public or private research centers.
L'archive ouverte pluridisciplinaire HAL, est destinée au dépôt et à la diffusion de documents scientifiques de niveau recherche, publiés ou non, émanant des établissements d'enseignement et de recherche français ou étrangers, des laboratoires publics ou privés. 


\title{
A Flexible Power Crossbar-based Architecture for Software-Defined Power Domains
}

\author{
Francesco Di Gregorio, Gilles Sassatelli, Abdoulaye Gamatié, Arnaud Castelltort \\ LIRMM, Univ Montpellier, CNRS \\ Montpellier, France \\ firstname.lastname@lirmm.fr
}

\section{Acknowledgments}

This work was carried out as part of the ICARE project funded by the Occitanie region, France.

\section{Keywords}

$\ll$ Smart grids $\gg, \ll$ Microgrid $\gg, \ll$ Distributed Power $\gg, \ll$ Energy system management $\gg, \ll$ SoftwareDefined Power Domains $\gg$.

\begin{abstract}
This paper proposes a novel approach referred to as "Software-Defined Power Domains", relying on a power crossbar component that makes it possible to setup arbitrary electrical topologies onto rather densely connected physical micro-grids. It presents the proposed crossbar architecture together with its topology switching operation and efficiency analysis.
\end{abstract}

\section{Introduction}

Energy transition and design of sustainable energy systems will require incremental yet profound longterm structural changes in energy production, storage and delivery. Besides the obvious expected role of renewable energies as sources, one prominent challenge lies in finding solutions capable of supporting the required gradual changes to power grids, from centralized generation towards distributed, heterogeneous and hybrid systems [1]. Smart grids and much of the work conducted in that area are meant to mature a portfolio of technologies enabling the advent of resilient self-regulated multi-scale power infrastructures capable of optimizing the overall efficiency and lifting dependence on fossil fuel energies.

Distributed generation exploiting intermittent renewable energy sources, distributed energy storage and smart scheduling decrease power losses by favoring the use of locally produced or stored energy, thereby significantly decreasing the average energy travel distance as well as the number of required current/voltage conversions. The main challenges are then to design technologies and control systems amenable to the integration into existing grids whatever their nature. We regard flexibility, in particular in terms of energy transport, as a primary concern that requires specific attention.

This paper therefore proposes a novel DC micro-grid (DCMG) crossbar architecture that makes it possible to install time-changing electrical topologies onto the existing physical architectures. We improve the state-of-the-art by proposing a system organization centered around a power crossbar that enables software-defined electrical topologies with the corresponding digital control architecture. This enables to handle dynamic and transparent switching (handover operation) from one set of energy actors to another. Our approach provides notable advantages through its flexibility, such as minimization of distribution / conversion losses via selection of appropriate routes between sources and loads. As the digital control architecture performs constant, high-frequency monitoring of multiple voltages and currents, the approach enables to setup a reactive failure detection and mitigation capable of recovering from various failures without interruption of energy delivery. 


\section{Related Works \\ DCMG Architectures}

The choice of the architecture is very important in the design of DCMG systems [5] [6] [7] [10]. It influences many factors such as the cost, resiliency, controllability, reliability, availability, resource utilization, and flexibility. DCMG architectures have been surveyed according to the number of available paths between loads and the AC grid in [6]. In this survey, it is shown that system reliability heavily depends on path diversity: in radial and ring (see Fig. 1.b) configurations for example, there is a single AC feeder. The architecture topology then exhibits more or less paths to the AC grid interface. The limitation of these architectures is in case a fault occurs on the AC feeder, the whole micro-grid functionality will be compromised because of the impossibility to access this always present energy resource, thereby decreasing reliability.

To overcome these limitations, interconnected architectures such as ladder-type [10] and zonal-type DCMG [9] can be used. These two architectures solve the reliability issue by adding AC feeders and additional redundant paths to the power network. However, this addition increases the architecture implementation costs and decreases the resource utilization because of the rather static [8] [10] nature of most current architectures. To enhance the architecture flexibility, other redundant paths are usually added to the system to connect a point $\mathrm{A}$ with a remote point $\mathrm{B}$, i.e. a load with the AC feeder. This adds complexity and costs to the whole system. In this paper, we use the term Energy Player (EP) to denote all energy devices involved in a micro-grid including distributed energy resources (DERs), loads, Energy Storage Systems (ESS) and AC grid. Even though the concept of energy routing already exists in literature [4] [3], there is no, to the best of our knowledge, physical implementation of a device which enables to proactively route the energy between EPs.

The proposed power crossbar-based architecture aims to tackle these issues by adding the possibility to dynamically and electrically reconfigure the power network by software. This greatly enhances the flexibility of the system compared to the existing static architectures. Moreover, power crossbars enable energy routing through the power network and increase the number of redundant paths between loads and the AC feeder, improving reliability without compromising resource utilization. Finally, this architecture further enables dynamic topology switching, opening an avenue for various run-time optimizations such as minimizing distribution losses or increased resilience through instant failure detection and recovery.

\section{Crossbar Architectures}

The architecture of an energy router is proposed in [15] which interfaces 2 AC Microgrids (ACMG) to the electrical grid. This energy router controls the power flow between the three ports using power converters. The limitation of this configuration is that it is static and not scalable. In fact, the greater the number of ports, the higher the complexity in its control. Moreover, this architecture has a single point of failure. In fact, a fault on the common DC Bus (DCB) determines the inability to interconnect the three ports.

Data crossbars as found in telecommunication networks are on their side specifically designed to create independent point to point connections between a set of inputs and a set of outputs. In a crossbar architecture, each input is connected to a dedicated bus which can be connected to any output bus by using one of the switches present at every X-Y cross-point. This allows to dynamically create data path where information flow from the input to the output. This technique is well known in computer science as circuit switching [16]. The advantage of this architecture is that a failure on a bus does not compromise the functioning of the whole crossbar, but only of the connected units. Such crossbars are however oriented, therefore limiting the applicability should one draw inspiration from such communication crossbars towards a power switching architecture where input and output concepts are irrelevants. Indeed a connection between two "outputs" is possible at the cost of allocating an input bus, thereby limiting the flexibility of the architecture.

The proposed crossbar architecture favours connections between EP groups and lifts this limitation. Each EP has the possibility to select among $M$ independent busses (see Fig. 3.b) of the local crossbar which, 
Table I: Comparison of crossbar architectures.

\begin{tabular}{c|ccc}
\hline Architecture & Cost & Flexibility & Reliability \\
\hline Energy Router in [15] & Low & Low & Low \\
Crossbar (communication network) & High & Medium & Medium \\
Crossbar (proposed architecture) & Medium & High & High \\
\hline
\end{tabular}

in turns, can connect to the busses of the adjacent crossbars. This permits to dynamically create, destroy and bind independent groups of EPs which share energy without assumption on power flow direction. Moreover, the proposed architecture is more reliable because a failure on a DCB does not compromise the functioning of any of the connected EPs which still can exploit the $M-1$ remaining busses of the crossbar. Table I qualitatively compares the three architectures in terms of cost, flexibility and reliability.

\section{Crossbar-based DCMG Architecture}

The architecture of the proposed crossbar-based DCMG is shown in Fig. 1.c. It consists of a set of DERs (i.e. solar panel, wind pole), loads (i.e. servers, electric vehicles etc.) and ESSs (i.e. batteries or super-capacitors) interconnected together by using power crossbars. In order to let this heterogeneous set of energy sources and loads interact together, power converters are used. Fig. 2 shows the structure of the conventional bidirectional DC/DC converter used to interface all the EPs. Buck / Boost modes are supported in both directions thanks to the symmetrical structure. The power crossbar, as will be explained in the next section, guarantees galvanic isolation for each EP. Then, it allows the dynamic reconfiguration of the DCMG by software. Indeed, it permits to dynamically create, decompose or even connect independent clusters of EPs, here referred as Power Domains (PDs). It allows resource sharing between sources and loads connected to different crossbars. Finally, it acts as a bridge in order to connect any two adjacent power crossbars without involving the local EPs. These EPs can still share their local resources by using an alternative independent bus of the crossbar to which they are physically connected.

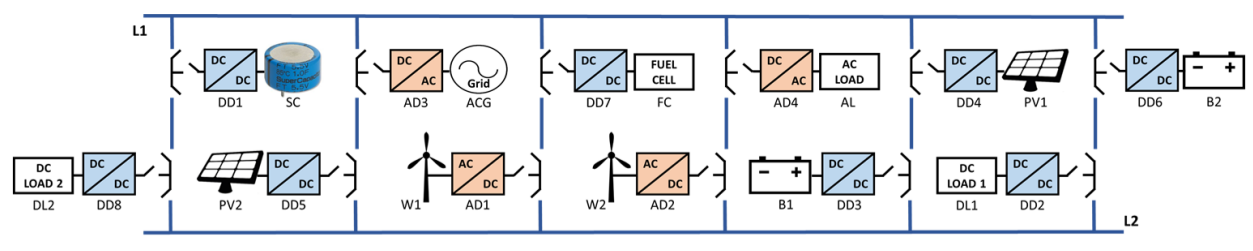

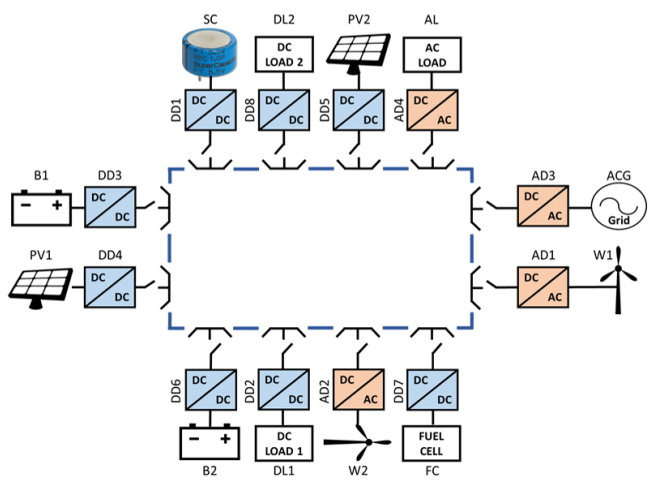

b) Ring

a) Ladder

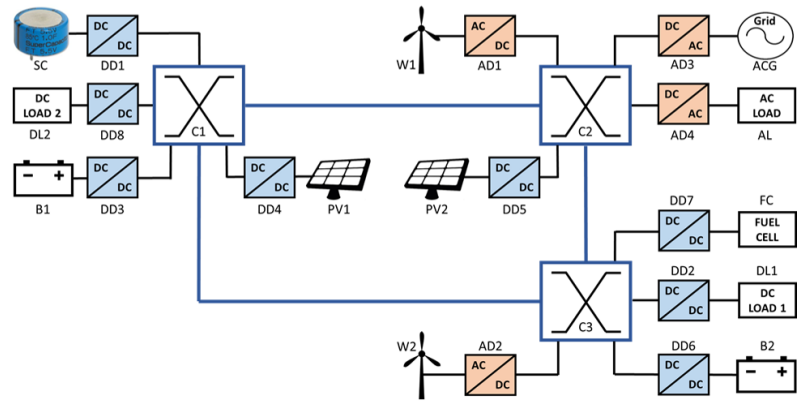

c) Our proposal: Crossbar

Fig. 1: DCMG implementation example: a) Ladder, b) Ring, and c) Crossbar-based architectures.

The control of the whole system and its associated crossbar is assigned to one or more Systems-onChip (SoCs) integrated on each crossbar. In particular, the Master-Slave control technique [2] [10] is used for the local power management. In Master-Slave technique a Master controller is chosen with the objective to keep the voltage on the common DCB constant. All the other controllers of the same 


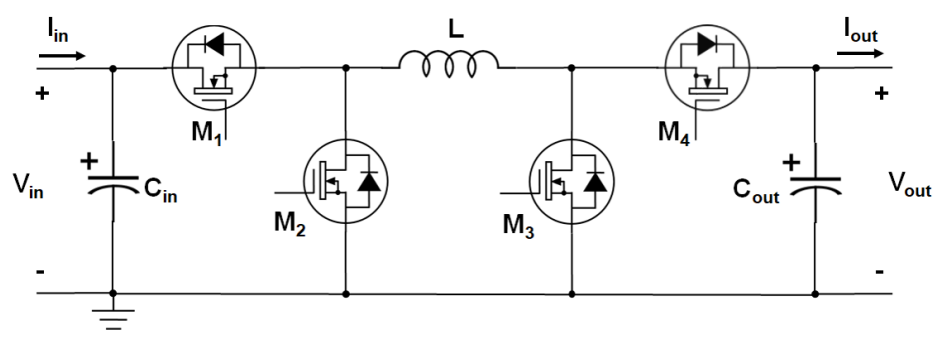

Fig. 2: Schematic of the Bidirectional DC/DC Converter configuration used in this work.

PD act as Slaves either injecting current into the DC Bus or plain loads. PI or PID controllers, which drive power converters in Constant Current (CC) or Constant Voltage (CV) mode, are implemented directly in software by exploiting the peripherals of the SoCs. Furthermore, diagnosis and fault recovery functionalities are handled by using local sensors and control. Finally, the addition of a communication interface allows different power crossbars to communicate, enabling the application of global and smart energy management strategies [8] to either increase the overall energy efficiency, reduce the energy cost or increase the reliability for critical loads, etc.

\section{Power Crossbar}

The power crossbar is the device that routes energy through the power network and defines PDs in software. It consists of a set of power switches (i.e. relays, MOSFET switches, etc.) which are controlled by software in order to physically define electrical paths on the Power Network for energy transfer between two or more EPs. Power crossbars have an arbitrary number of external ports and independent busses. Depending on this number, some connection combinations between external ports are achieved. The bigger the number of ports and independent busses, the greater the flexibility, at the expense of more complexity in the crossbar topology and control.

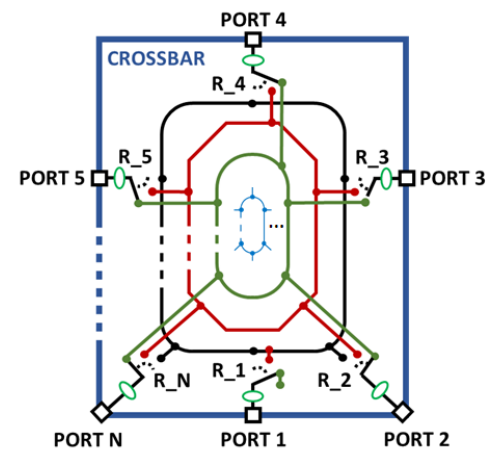

a) Top view

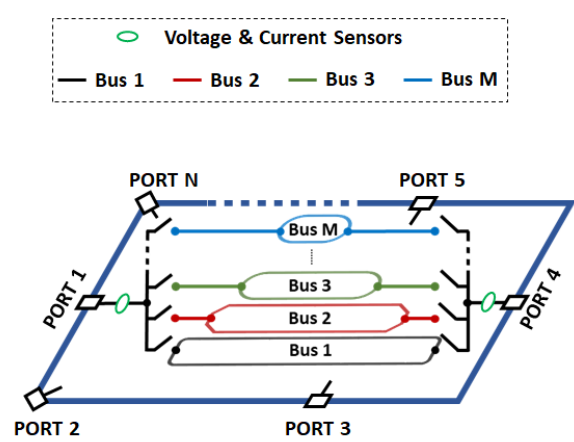

b) Side view

Fig. 3: N-ports, M-busses crossbar: a) Top and b) Side view.

Fig. 3 shows the architecture of an N-ports, M-busses power crossbar. Fig. 3.a represents a top view, outlining the number of ports while Fig. 3.b shows a side view, where the busses with their power switches are highlighted. The number of ports defines the maximum number of external connections of the crossbar. The number of busses defines both the maximum number of independent paths that can be created on a crossbar, and the maximum power capacity the crossbar can support. The maximum transmission power can be dynamically adjusted by connecting more busses in parallel.

\section{Implementation Costs}

Another important aspect to consider in the architecture is the implementation cost, notably in relation with the number of power switches. Here, we use the number of switches normalized to the number of EPs, denoted by $N_{S w E P}$, as a metric to estimate the implementation cost and compare different DCMG architectures. For instance, in Ring and Ladder ones (see Fig. 1.b and Fig. 1.a respectively), $N_{S w_{-} E P}$ is 
always equal to 3 . In the proposed DCMG architecture, assuming identical crossbar instances, $N_{S w_{-} E P}$ is calculated with the following formula (1):

$$
N_{s w E P}=M *\left(1+N_{C R I N T} / N_{E P}\right)
$$

where $\mathrm{M}$ is the number of independent busses of the crossbar, $N_{C R I N T}$ is the total number of crossbar ports used for interconnections, $N_{E P}$ is the total number of EPs in the DCMG. For instance, in Fig. 1.c $N_{C R I N T}=6$ and $N_{E P}=12$. From (1), using 2-busses crossbars $(M=2)$, one obtains an implementation cost of 3 switches per EP, which is equal to that of Ring and Ladder architectures. However, for our crossbar-based architecture, the cost can be reduced by reducing the ratio $N_{C R I N T} / N_{E P}$, which is obtained by adding more EPs per crossbar.

\section{Control Strategy}

Many control techniques for microgrids can be found in the literature [10][13][14]. A two-level hierarchical control has been chosen for the proposed crossbar-based DCMG. In particular, the first level of control manages the power balance between EPs connected to the same PD. Master-Slave technique is used to reach this objective for its simplicity and effectiveness in small and medium-sized power networks. Then, the second level of control defines PDs, assigns Master role to a power converter in each $\mathrm{PD}$ and manages the dynamic topology switching operation.

\section{Dynamic Topology Switching}

Having the ability to match sources to current load is here regarded as a prominent advantage. We here demonstrate the ability of performing dynamic (run-time) topology switching, which we refer to as handover operation.

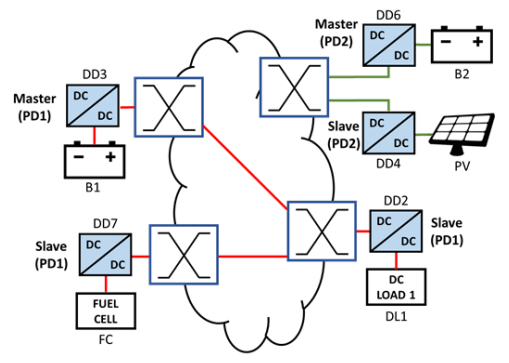

a) Init

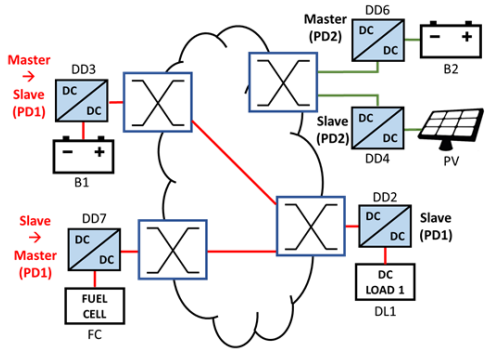

b) Handover

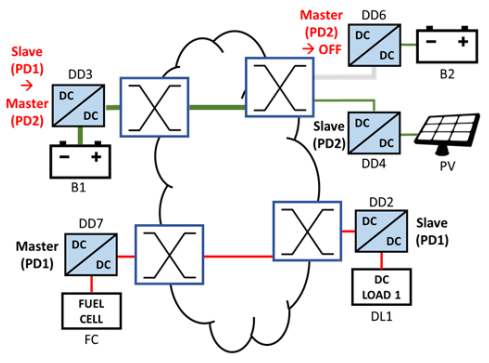

c) Domain change

Fig. 4: Dynamic topology switching protocol: a) Init, b) handover and c) power domain change.

This makes it possible to change the Master control of a PD. In a crossbar-based DCMG each PD requires a Master that may turn into a Slave after a handover operation. We here describe a reliable, experimentally validated technique for handover operation. The technique does not alter the power quality that we here define as maximum acceptable voltage ripple, arbitrarily set to $6 \%$. The method is simulated with the Powersim simulator. We consider the electromechanical relays that provide galvanic isolation for each EP. The simulated scenario, depicted in Fig. 4, involves two generic voltage sources of 20V, represented by a battery B1 and a fuel cell FC that reach a DC load DL1 by using the power crossbar network. At the beginning, the B1 converter DD3 is the Master of PD1, guarantying a constant voltage of $36 \mathrm{~V}$ on the DCB and FC converter DD7 is in slave mode helping feeding DL1. On the other domain PD2, B2 converter DD6 is the Master, keeping a constant voltage of $30 \mathrm{~V}$, while receiving the maximum power the solar panel PV, which is in Slave mode, provides. Then, the handover protocol permits to change the Master control of PD1 between the two sources and lets B1 change domain, switching from discharging mode to charging mode. 
Table II: Description of the dynamic topology switching steps.

\begin{tabular}{|c|l|l|}
\hline Time & \multicolumn{1}{|c|}{ Description } & \multicolumn{1}{c|}{ Comments } \\
\hline 0ms & Initial state. & $\begin{array}{l}\text { The initial configuration is depicted in } \\
\text { Fig. 4.a. }\end{array}$ \\
\hline $50 \mathrm{~ms}$ & $\begin{array}{l}\text { The DCMG controller gradually changes the } \\
\text { current set point of FC in order to reach the } \\
\text { current contribution of B1. }\end{array}$ & $\begin{array}{l}\text { This reduces oscillations during the handover } \\
\text { operation. }\end{array}$ \\
\hline $55 \mathrm{~ms}$ & $\begin{array}{l}\text { The handover operation takes place by revers- } \\
\text { ing the control of the Master B1 and the Slave } \\
\text { FC. Then, the re-configuration process starts } \\
\text { by setting relays. }\end{array}$ & $\begin{array}{l}\text { B1 current is set according to the last mea- } \\
\text { surement of its current sensor in order to re- } \\
\text { duce perturbations on the DCB. Note that } \\
\text { chosen relays [12] need 25ms to operate and } \\
\text { 5ms to release. }\end{array}$ \\
\hline $60 \mathrm{~ms}$ & $\begin{array}{l}\text { B1 set point is gradually brought to 0A. } \\
\text { This permits to disconnect B1 without per- } \\
\text { turbing the DCB of PD1. }\end{array}$ \\
\hline $80 \mathrm{~ms}$ & $\begin{array}{l}\text { Power relays close and the reconfiguration } \\
\text { process is completed. B2 disconnects from } \\
\text { the PD2 domain and is turned OFF so as to } \\
\text { let B1 charge and take all of the power com- } \\
\text { ing from the solar panel. Master control of } \\
\text { PD2 pass to B1. }\end{array}$ & $\begin{array}{l}\text { The controller of B1 adapts its input current in } \\
\text { order to keep the DCB to a constant voltage of } \\
\text { 30V. }\end{array}$ \\
\hline
\end{tabular}
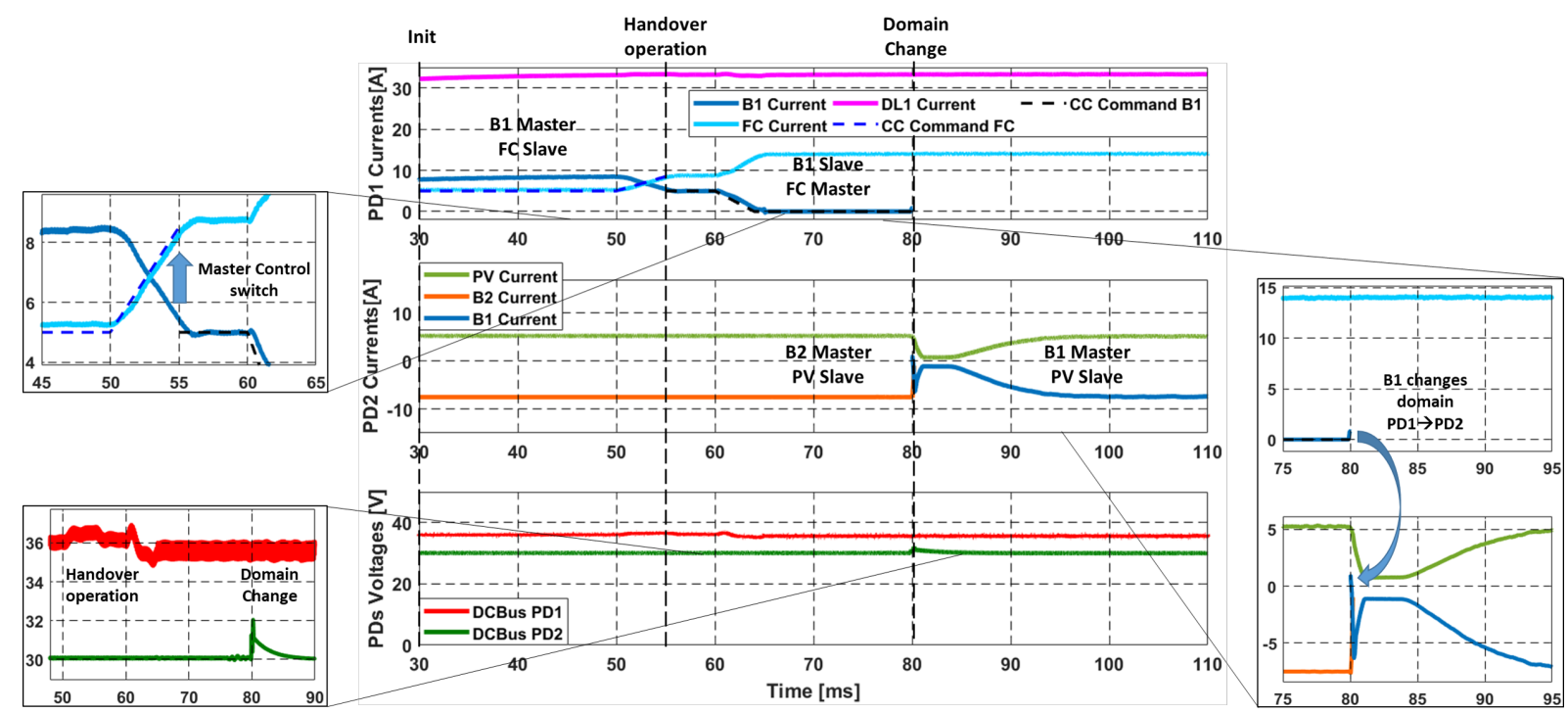

Fig. 5: PSIM simulation of handover and domain change.

The main steps of the handover and domain change operation are summarized in Table II and the simulation results depicted in Fig. 5. A ramp function with a slope of $2.6 \mathrm{~A} / \mathrm{ms}$ is used in the simulation to gradually change the set points to the PI controllers. As shown in Fig. 5 the handover operation does not affect the voltage of the PD1 DCB, which undergoes a slight voltage oscillation of $1 \mathrm{~V}$ corresponding to $2,78 \%$ of its nominal value. A greater voltage peak of $2 \mathrm{~V}$ corresponds to the activation of relays due to DD3 capacitances already charged from previous operation.

\section{Fault Handling}

The proposed DCMG architecture offers better fault handling capabilities with respect to the previous architectures. The presence of $\mathrm{M}$ independent busses per crossbar permits to overcome to fault conditions by simply switching the operating bus. Path diversity and dynamic reconfiguration permit to isolate the fault and to perform recovery by selecting another redundant path. Different types of fault can occur in 
a DCMG architecture. In this work, we consider two main types of fault:

- fault on a DCB (short circuit)

- fault on a terminal relay

Fig. 6 presents a schematic view of the presented architectures highlighting the interconnection components such as terminal relays, internal and external busses. A 2-busses 6-ports power crossbar has been chosen in this work which represents a good compromise between implementation costs and flexibility offered.

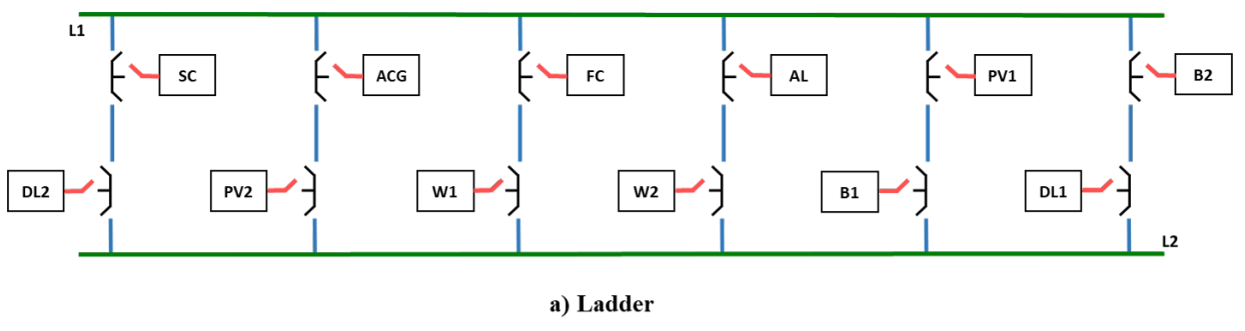

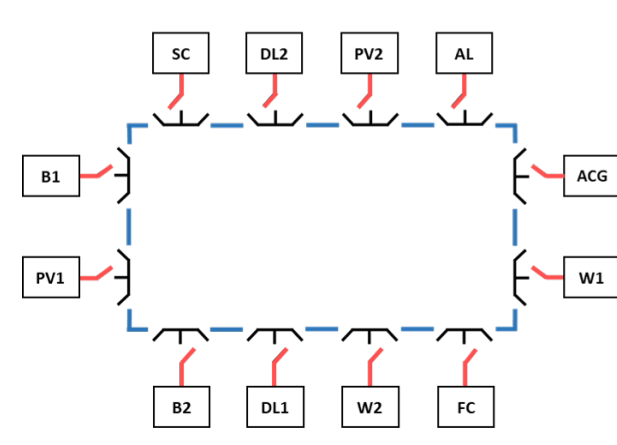

b) Ring

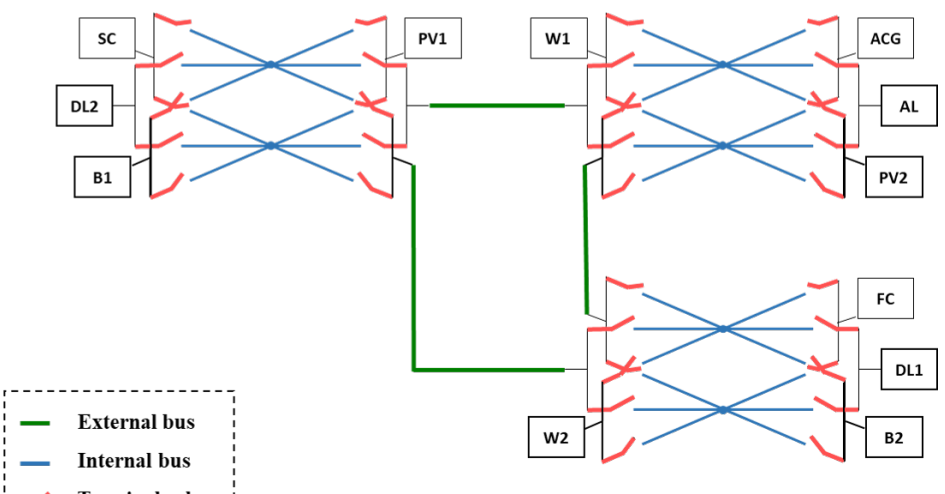

c) Our proposal: Crossbar

Fig. 6: Bus segmentation in the three DCMG architectures: a) Ladder, b) Ring and c) Crossbar-based.

A fault on a DCB can be more or less critical depending on its position. In ring architecture, for example, the bus is segmented along all the ring (see Fig. 6.b) and all the segments are equally critical. A fault on a segment does not have serious consequences, but two consecutive faults highly limit the connectivity of the EPs.

On the other hand, ladder architecture presents two types of busses (see Fig. 6.a): L1 / L2 external busses and the internal busses in each step of the ladder. Faults on the external busses are more critical especially when the DCMG scale up. In fact, two consecutive faults on the two external busses highly affect the connectivity of the whole DCMG.

Finally, the proposed crossbar-based DCMG presents two types of busses (see Fig. 6.c): internal busses which permit the connection of the EPs attached to the same crossbar and external busses allowing the interconnection between crossbars. A fault on an external bus is less critical than internal busses. In fact, consecutive faults on the external busses of the same crossbar limits the connection of the local EPs to the other crossbars. However, they still have the possibility to exchange energy locally. A fault on an internal bus does not compromise the functioning of any EP which can use the others $M-1$ busses to interconnect. Consecutive faults on the busses of the same crossbar can limit the connectivity of the EPs connected to that crossbar but the others EPs can still function properly. Moreover, consecutive faults on busses of different crossbars does not compromise any of the EPs.

Terminal relays are the most critical parts in a connection between two EPs. In fact, a fault on these makes it impossible to access the power network by the associated EP. While ring and ladder architectures have a single terminal relay per EP, the proposed crossbar-based architecture has M terminal relays per EP. This redundancy permits an higher level of reliability for the crossbar-based architecture with respect to 
the others. To summarize, the bus segmentation redundancy and the dynamic topology reconfiguration offered by the crossbar-based DCMG provides better fault handling capability as well as fault isolation and recovery.

\section{Efficiency Analysis}

We analyze and compare the energy-efficiency of the three architectures shown in Fig. 1. To estimate the energy transport efficiency, the number of crossed relays is considered. We assume that cable losses can be neglected due to short distance and only account for the contribution to the transmission losses given by the static power loss needed to activate a relay and the power losses contact resistance. Unit loss figures are extracted from a commercial power relay for ensuring realistic assessment even though relative units such as number of relays crossed would yield to similar conclusions. The chosen relay [12] has a static power consumption of $1.7 \mathrm{~W}$, a maximum DC current of $50 \mathrm{~A}$ and uses $\mathrm{AgSnO} 2$ as contact material that presents an average contact resistance of $2 \mathrm{~m} \Omega$ [11]. The comparison is made by analyzing the shortest paths allowing to connect a pair of source-load. Then, depending on the number of relays found on that path, an estimation of transmission efficiency is made.

Table III: Shortest paths ranking for the source-load pair B1 $\rightarrow$ DL1.

\begin{tabular}{|c|c|c|c|}
\hline $\begin{array}{c}\text { Shortest } \\
\text { Path }\end{array}$ & Path Ring & Path Ladder & Path Crossbar-based \\
\hline 1st & $\mathrm{B} 1 \rightarrow \mathrm{PV} 1 \rightarrow \mathrm{B} 2 \rightarrow \mathrm{DL} 1$ & $\mathrm{~B} 1 \rightarrow \mathrm{L} 2 \rightarrow \mathrm{DL} 1$ & $\mathrm{~B} 1 \rightarrow \mathrm{C} 1(1) \rightarrow \mathrm{C} 2(1) \rightarrow \mathrm{DL} 1$ \\
\hline 2nd & $\begin{array}{c}\mathrm{B} 1 \rightarrow \mathrm{SC} \rightarrow \mathrm{DL} 2 \rightarrow \mathrm{PV} 2 \rightarrow \mathrm{AL} \rightarrow \\
\mathrm{ACG} \rightarrow \mathrm{W} 1 \rightarrow \mathrm{FC} \rightarrow \mathrm{W} 2 \rightarrow \mathrm{DL} 1\end{array}$ & $\mathrm{~B} 1 \rightarrow \mathrm{PV} 1 \rightarrow \mathrm{L} 1 \rightarrow \mathrm{B} 2 \rightarrow \mathrm{DL} 1$ & $\mathrm{~B} 1 \rightarrow \mathrm{C} 1(2) \rightarrow \mathrm{C} 2(1) \rightarrow \mathrm{DL} 1$ \\
\hline 3rd & Does not exist. & $\begin{array}{l}\mathrm{B} 1 \rightarrow \mathrm{L} 2 \rightarrow \mathrm{W} 2 \rightarrow \mathrm{AL} \rightarrow \mathrm{L} 1 \rightarrow \\
\mathrm{B} 2 \rightarrow \mathrm{DL} 1\end{array}$ & $\mathrm{~B} 1 \rightarrow \mathrm{C} 1(1) \rightarrow \mathrm{C} 2(2) \rightarrow \mathrm{DL} 1$ \\
\hline
\end{tabular}

In Table III, an example of comparison between the three architectures is made, by considering the pair battery B1 with the DC load DL1. In particular, the table presents the three shortest paths allowing to connect B1 with DL1. Note that the ring architecture always has two paths to connect a source with a load whose sum equals the number of serial relays on the ring. Ladder architecture has a better organization of its resources thanks to its two common busses L1 and L2 which overall provide higher efficiency and path redundancy. Crossbar-based architecture, thanks to the bus segmentation and configurability makes for a higher number of redundant paths with higher efficiency.

Fig. 7 shows an estimation of transmission efficiency obtained in the three architectures. In particular, four samples of source-load pairs were selected and represented, next to the average on all possible pairs, represented in the fifth columns. Results show that the crossbar-based architecture has a higher energy efficiency compared to Ring and Ladder. A transmission power of $1051 \mathrm{~W}$ is used for the simulation, which corresponds to the optimal transmission power calculated from the relay characteristics: static power losses in the coil and contact resistance.

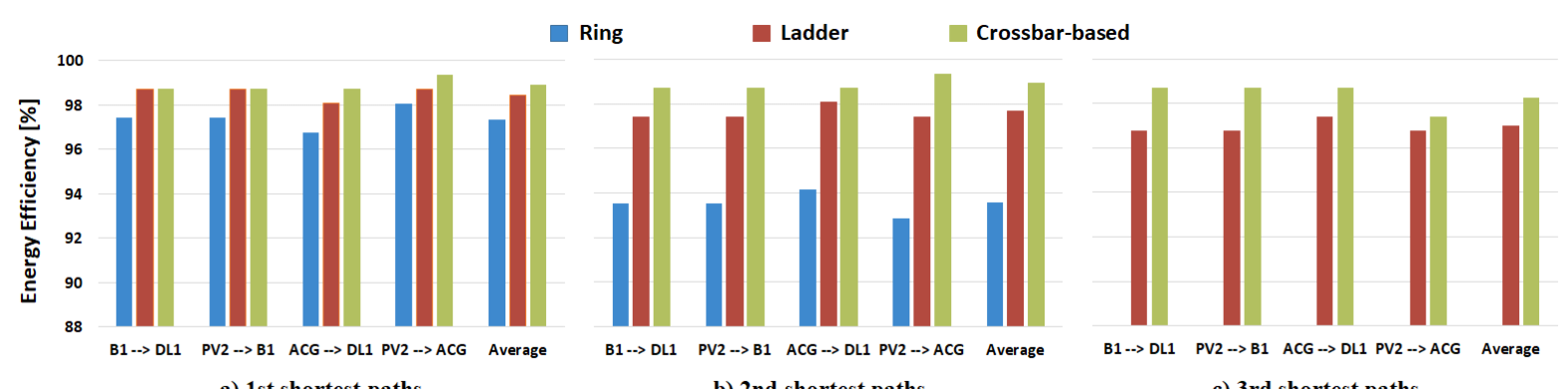

Fig. 7: Energy efficiency comparison between Ring, Ladder and Crossbar-based architectures for several source-load pairs: a) 1st, b) 2nd and c) 3rd shortest paths. 


\section{Conclusion}

Energy transition is imminent and the role of renewable energy sources will be fundamental. The heterogeneity and intermittent nature makes static topology / architectures unsuited. Dynamic, softwaredefined architectures should be considered to facilitate this transition. This paper proposes a novel approach referred to as "software-defined power domains" that relies on an energy crossbar component. The solution makes it possible to setup arbitrary electrical topologies onto densely connected physical micro-grids. We improve state-of-the-art by proposing a system organization centered around this crossbar that enables software-defined electrical topologies with the corresponding digital control architecture that handles dynamic and transparent handover from one set of energy actors to another. This approach provides distinct advantages thanks to its flexibility, such as minimization of distribution / conversion losses through selection of appropriate routes between sources and loads. It provides a greater reliability, resource utilization and resilience. Finally, the dynamic switching operation together with an efficiency analysis of the proposed architecture are covered in the paper. Measures of the handover operation are underway on a recently realized prototype of crossbar-based DCMG.

\section{References}

[1] R. Salas-Puente, S. Marzal, R. Gonzlez-Medina, E. Figueres, and G. Garcer: Efficient management strategy of the power converters connected to the DC bus in a hybrid microgrid of Distributed Generation, EPE 2017-ECCE Europe, pp. P.1-P.10

[2] D. E. Olivares et al.: Trends in Microgrid Control, IEEE Transactions on Smart Grid, Vol. 5 no 4, pp. 19051919, July 2014

[3] M. H. Amini, K. G. Broojeni, T. Dragievi, A. Nejadpak, S. S. Iyengar, and F. Blaabjerg: Application of cloud computing in power routing for clusters of microgrids using oblivious network routing algorithm, EPE 2017-ECCE Europe, pp. P.1-P.11

[4] A. Brocco: Fully distributed power routing for an ad hoc nanogrid, 2013 IEEE International Workshop on Intelligent Energy Systems (IWIES), pp. 113-118, 2013

[5] D. Burmester, R. Rayudu, W. Seah, and D. Akinyele: A review of nanogrid topologies and technologies, Renewable and Sustainable Energy Reviews, Vol. 67, pp. 760-775, 2017

[6] D. Kumar, F. Zare, and A. Ghosh: DC Microgrid Technology: System Architectures, AC Grid Interfaces, Grounding Schemes, Power Quality, Communication Networks, Applications, and Standardizations Aspects, IEEE Access, Vol. 5, pp. 12230-12256, 2017

[7] D. Magdefrau, T. Taufik, M. Poshtan, and M. Muscarella: Analysis and review of DC microgrid implementations, 2016 International Seminar on Application for Technology of Information and Communication (ISemantic), pp. 241-246, 2016

[8] M. Gunasekaran, H. Mohamed Ismail, B. Chokkalingam, L. Mihet-Popa, and S. Padmanaban: Energy Management Strategy for Rural Communities DC Micro Grid Power System Structure with Maximum Penetration of Renewable Energy Sources. Applied Sciences 2018, Vol. 8, no. 4, pp. 585

[9] E. Tironi, M. Corti, and G. Ubezio: Zonal electrical distribution systems in large ships: Topology and control, 2015 AEIT International Annual Conference (AEIT), pp. 1-6, 2015

[10] J. Kumar, A. Agarwal, and V. Agarwal: A review on overall control of DC microgrids, Journal of Energy Storage, Vol 21, pp. 113-138, 2019

[11] A. Ksiazkiewicz, G. Dombek, and K. Nowak: Change in Electric Contact Resistance of Low-Voltage Relays Affected by Fault Current, Materials (Basel, Switzerland) Vol. 12, pp. 1-11, July 2019

[12] Finder: High Power relay 50 A 67 Series, 67.23.9.012.4300 datasheet, 2016

[13] P. Borazjani, N. I. A. Wahab, H. B. Hizam, and A. B. C. Soh: A review on microgrid control techniques, 2014 IEEE Innovative Smart Grid Technologies - Asia (ISGT ASIA), Kuala Lumpur, pp. 749-753, 2014

[14] J. M. Guerrero, M. Chandorkar, T. Lee and P. C. Loh: Advanced Control Architectures for Intelligent MicrogridsPart I: Decentralized and Hierarchical Control, IEEE Transactions on Industrial Electronics, Vol. 60 no 4, pp. 1254-1262, April 2013

[15] Y. Liu, Y. Fang, J. Li: Interconnecting Microgrids via the Energy Router with Smart Energy Management, Energies, Vol. 10 no 9, pp. 1297, 2017

[16] G. Broomell and J. R. Heath: Classification Categories and Historical Development of Circuit Switching Topologies. ACM Comput. Surv., Vol. 15 no 2, pp. 95133, 1983 